\title{
LR White is preferable to Unicryl for immunogold detection of fixation- sensitive nuclear antigens
}

\author{
V.V. Philimonenko1, J. Janáček³, and P. Hozák ${ }^{1,2}$
}

${ }^{1}$ Institute of Experimental Medicine, Department of Cell Ultrastructure and Molecular Biology, Academy of Sciences of the Czech Republic, Vídeňská 1083, 14220 Prague 4 - Krč, Czech Republic; ${ }^{\text {rd }}$ Medical Faculty, Department of Cell and Molecular Biology, Charles University, Ruská 87, 10000 Prague 10, Czech Republic; ${ }^{3}$ Department of Biomathematics, Institute of Physiology, Academy of Sciences of the Czech Republic, Vídeňská 1083, 14220 Prague 4 - Krč, Czech Republic

Accepted: 12/04/02

Key words: cell nucleus, ultrastructure, immunogold labeling, acrylic resins

\section{SUMMARY}

The purpose of this study was to compare two electron microscopy embedding media - LR White and Unicryl - with regard to cell morphologyical and immunohistochemical preservation properties for the study of fixation-sensitive nuclear antigens. Human cervical carcinoma (HeLa) cells were fixed with $2 \%$ paraformaldehyde and $0.1 \%$ glutaraldehyde, and embedded in parallel in the two resins: LR White and Unicryl using; two different polymerization protocols were used for each resin. Preservation of fine nuclear structure was good after LR White and poor after Unicryl embedding. Immunogold labeling of Sm antigen was significantly stronger on LR White sections. Polymerization by UV light resulted in stronger and more specific labeling than heat polymerization. These results show that LR White is advantageous over Unicryl for the study of nuclear antigens requiring delicate aldehyde fixation.

\section{INTRODUCTION}

Immunoelectron microscopy has been widely used to determine the intracellular distribution of specific biochemical components to gain information on their function. Adequate preservation of ultrastructure and antigenic reactivity of a biological specimen depends on a combination of factors, the most important of which are the method of fixation, the technique of tissue processing, the choice of embedding agent, and the way of resin polymerization. As many epitopes are sensitive to high concentrations of glutaraldehyde (Miller, 1972; Smit et al., 1974), a gentle fixation with a mixture of $2-4 \%$ formaldehyde with $0.1 \%-1.0 \%$ glutaraldehyde is generally recommended as a good compromise for both ultrastructure and antigenicity preservation (Tokuyasu, 1984). During embedding, exposure of the tissue to extracting agents and denaturing temperatures should be minimized. This, in turn, can be achieved only if the embedding medium has low viscosity allowing fast infiltration even at lower temperatures, and tolerates small amounts of water, which helps to avoid drastic complete dehydration of the sample. For subsequent immunolabeling on ultrathin sections, lightly cross-linked and hydrophilic resin facilitates access of the labeling agent to the epitope. A wide range of acrylic resins meeting these criteria is available (see e.g. Stirling, 1990). One of

Correspondence to: P. Hozák 
the most widely used is LR White, introduced in early 80-s and well described in both scientific and commercial literature (Newman and Hobot, 1987; Newman, 1999). Unicryl, initially under the name of Bioacryl, was formulated and described by Scala et al. in 1992. Embedding in this resin resulted in good ultrastructural preservation of the tissue and provided strong specific immunolabeling of the sections (Scala et al., 1992; Bogers et al., 1996; Goping et al., 1996). However, only two direct comparisons of LR White and Unicryl have been performed up to date with controversial results - for mRNA in situ hybridization and simultaneous localization of the protein product (Slater and Mason, 1994), and for the detection of several cytoplasmic enzymes and membrane proteins in chromaffin cells (Goping et al., 1996).

In this study, we have compared LR White and Unicryl for their suitability in studies of nuclear antigens. Fine structure preservation of cellular structures and density of immunogold labeling of $\mathrm{Sm}$ antigen were evaluated. Sm antigen represents a group of proteins participating in a variety of RNA processing events (see e.g. Will and Luhrmann, 1997; Seto et al., 1999) and was chosen as a typical and abundant nuclear antigen. The results show that after a mild aldehyde fixation and standard embedding procedure, better ultrastructure and stronger immunogold signal are achieved with the LR White resin. However, in cases with extremely low labelling efficiency Unicryl may provide more specific immunogold labeling. For both resins, polymerization by UV light is preferable to thermal polymerization.

\section{MATERIALS AND METHODS}

\section{Cell culture}

Human cervical carcinoma (HeLa) cells were grown at $37^{\circ} \mathrm{C}$ in suspension culture in Eagle minimum essential medium (S-MEM, Sigma-Aldrich, Prague, Czech Republic) supplemented with 5\% $(\mathrm{v} / \mathrm{v})$ foetal calf serum

\section{Antibodies}

Human autoimmune serum containing high titer of anti-Sm antigen antibodies (ANA Human Reference Serum \#5, Centers for Disease Control, Atlanta, USA) was used in dilution 1:500 and goat anti-human IgG antibodies conjugated with $10 \mathrm{~nm}$ colloidal gold particles (British BioCell International Ltd., Cardiff, UK) were used in dilution 1:50.

\section{Fixation and embedding for electron microscopy}

Cells were fixed for $40 \mathrm{~min}$ in $2 \%$ paraformaldehyde and $0.1 \%$ glutaraldehyde in Sörensen buffer (0.1 M sodium/potassium phosphate buffer, $\mathrm{pH} 7.3$; $\mathrm{SB})$ at room temperature, washed in two changes of SB (10 minutes each), incubated with $0.02 \mathrm{M}$ glycine in SB for $10 \mathrm{~min}$, washed again in SB and embedded in LR White (Polysciences Inc., Warrington, USA) or Unicryl (British BioCell International, Ltd., Cardiff, UK) according to standard protocol recommended by manufacturer. Briefly, cells were quickly dehydrated in series of pre-cooled ethanol solutions (30, 50, 70, 96, 100\%; 10 minutes each), the ethanol was then replaced with a mix of ethanol and resin $2: 1$ for 20 minutes, followed by ethanol/resin 1:2 mix for 20 minutes, and pure resin for 1 hour. Samples were infiltrated overnight with a fresh portion of pure resin at $4^{\circ} \mathrm{C}$, next day incubated for two hours with a fresh resin, and the resins were polymerized for 48 hours either at $4{ }^{\circ} \mathrm{C}$ under UV light (UV), or at $50^{\circ} \mathrm{C}(\mathrm{T})$. The whole procedure was performed at $0^{\circ} \mathrm{C}$ unless otherwise stated.

\section{Postembedding immunolabeling}

Thin sections ( $80 \mathrm{~nm}$ thick) were cut with a diamond knife (Diatome, Biel, Switzerland) on a Reichert Ultracut E (Leica Microsystems AG, Wetzlar, Germany) and mounted on 200 mesh gilded copper grids (Polysciences Inc., Warrington, USA). Non-specific labeling was blocked by preincubation with $10 \%$ normal goat serum (NGS, British BioCell International Ltd., Cardiff, UK), in PBTB (1\% BSA and $0.1 \%$ Tween 20 in PBS, pH 7.4) for 30 minutes at room temperature. The sections were then incubated for 1 hour with primary antibodies diluted in PBTB containing $1 \%$ NGS or only with PBTB with NGS for control, washed in three ten-minute changes of PBT $(0.005 \%$ Tween 20 in PBS), incubated 45 minutes with secondary gold-conjugated antibodies in PBTB, washed again twice in PBT, then twice in bidistilled water (10 minutes each wash), and air-dried. Finally, sections were contrasted with a saturated solution of uranyl acetate in water for $4 \mathrm{~min}$.

\section{Quantification of the gold labeling}

Immunostained sections were observed in a Philips CM100 electron microscope (Philips, Einthoven, The Netherlands) equipped with a CCD camera 
(model 673, Gatan, Pleasanton, USA) and 50 random digital electron microscope images of nuclear sections were taken per each experimental and control group. The area of nucleoplasm and the number of gold particles were measured on the images using a macro developed for LUCIA image-processing software (Laboratory Imaging Ltd., Prague, Czech Republic), and the density of gold labeling was calculated. Differences between samples were statistically evaluated by t-test.

\section{RESULTS}

We compared the ultrastructure and labeling intensity of Sm antigen in HeLa cells that were embedded in either LR White or Unicryl polymerized either at $50^{\circ} \mathrm{C}$ (LR-T, Uni-T) or at $4^{\circ} \mathrm{C}$ under UV light (LR-UV, Uni-UV). LR White blocks were easier to cut, and the ultrathin sections had higher affinity to uranyl acetate. Electron micrographs are shown in Fig. 1. Cytoplasmic structures
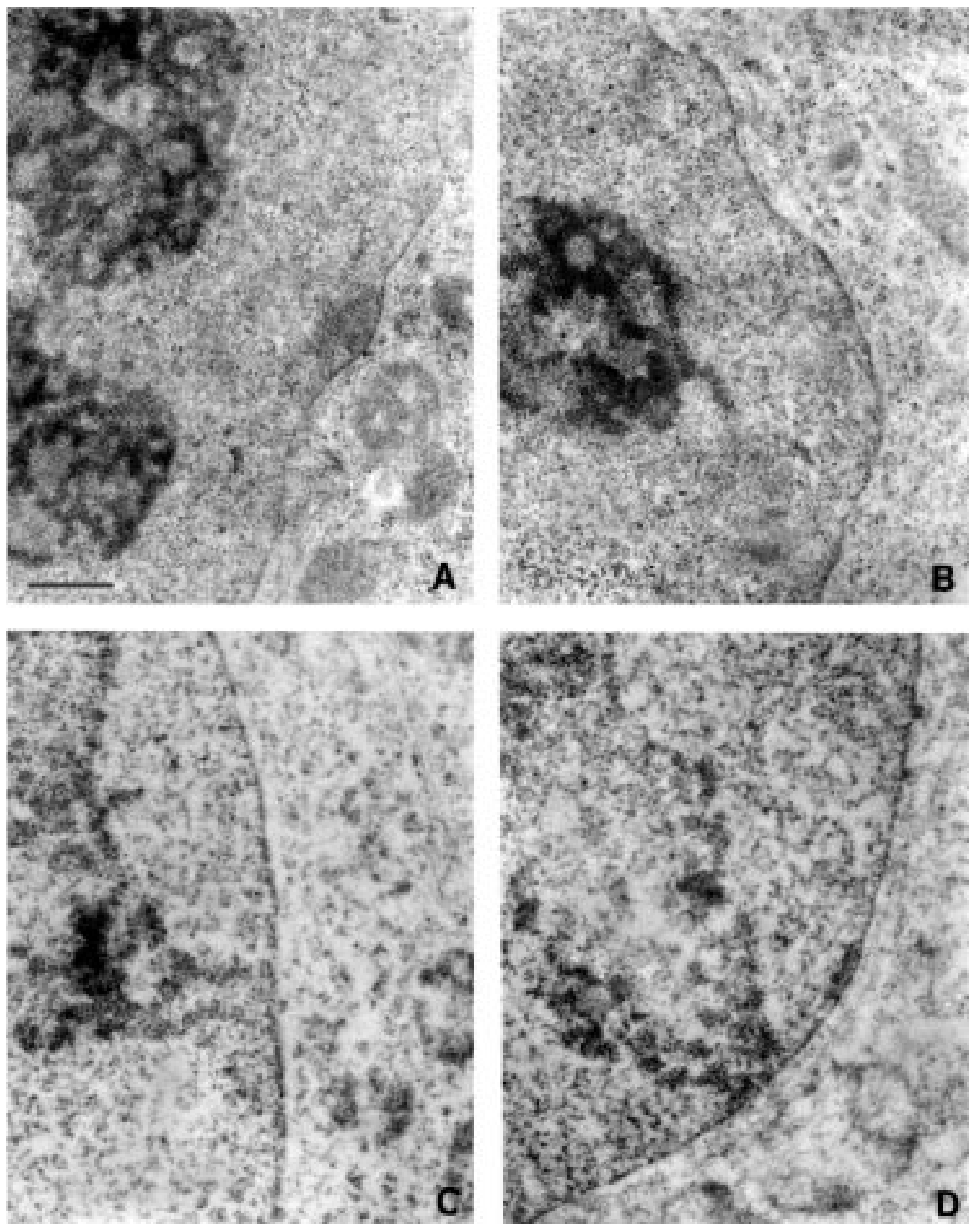

Fig. 1 - Electron micrograph of HeLa cell embedded in LR White (A, polymerization at $50^{\circ} \mathrm{C}$; $\mathrm{B}$, polymerization at $4^{\circ} \mathrm{C}$ under UV light), or in Unicryl (C, polymerization at $50^{\circ} \mathrm{C}$; D, polymerization at $4{ }^{\circ} \mathrm{C}$ under UV light). Bar, $500 \mathrm{~nm}$. 
and membranes were preserved to similar extent in all samples. Nucleoplasm and chromatin, however, had finer and more defined structure in LR Whiteembedded samples. After Unicryl embedding, nuclear interior appeared like rough, loosened meshwork of equal density throughout the nucleus, without clear distinction between euchromatin and heterochromatin domains. Nucleolar components - fibrillar centers, dense fibrillar component, and granular component - were well defined on LR White sections, while in Unicryl-embedded cells they were often indistinguishable. The method of resin polymerization had no prominent effect on the ultrastructure when LR White was used, but for Unicryl heat polymerization resulted in much worse morphology of the samples than polymerisation by UV light.

To compare the effectiveness of immunodetection of nuclear antigens, the density of postembedding immunogold labeling of Sm antigen was quantified. The results are presented in Fig. 2A. The labeling density of LR White-embedded samples is about two-fold higher than that of Unicryl-embedded specimens; the difference is statistically significant at $1 \%$ level (Table I). When comparing UV- and heat polymerization, there is no significant difference in the labeling density for Unicryl, while on LR White sections labeling density is higher by $18 \%$ after UV-polymerization than after thermal polymerization.

In order to compare the resins with respect to nonspecific binding of immunoreagents, the density of background labeling was quantified after omitting the primary antibodies (Fig. 2B). The background labeling density equaled $1.07 \%$ of the total labeling density for Uni-T, $0.65 \%$ for Uni-UV, $1.25 \%$ for LR$\mathrm{T}$, and $0.74 \%$ for LR-UV. The difference between samples was statistically non-significant - thus, nonspecific labeling was negligible in all cases. Nevertheless, the ratio between total labeling intensity and background label density is slightly better for Unicryl-embedded material (exceeds that for LR White-embedded cells by cca $15 \%$ (Table II)). For both resins, this ratio was by $40 \%$ higher when polymerization was performed at $4^{\circ} \mathrm{C}$ under UV light when compared to thermal polymerization.

These results demonstrate that the use of LR White is advantageous over Unicryl for the study of fixation-sensitive nuclear antigens as both cell ultrastructure and antigenic reactivity were better preserved.

\section{DISCUSSION}

In this study, HeLa cells embedded in LR White and Unicryl were compared with respect to ultrastructural preservation of cell structures and to the efficiency ofa nuclear antigen detection using postembedding immunoelectron microscopy. Cells were fixed with $2 \%$ formaldehyde in presence of and a minimal concentration $(0.1 \%)$ of glutaraldehyde $(0.1 \%)$ to) to maintain morphological pattern but still allow good antigen preservation. A standard embedding protocol was used; both resins are equally simple to use.

Embedding in LR White resulted in satisfactory morphology of cytoplasm and in good fine nuclear structure. This was expected, as we had used LR White for studies on nuclear structure for a long time with good results (see e.g. Hozak et al., 1994a;

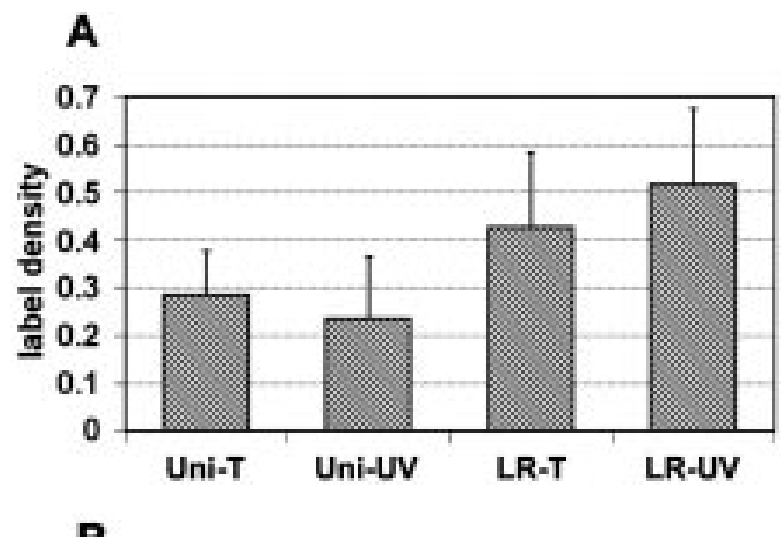

B

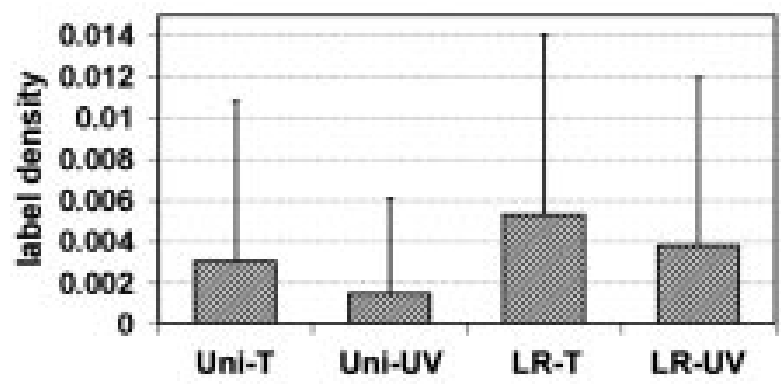

Fig. 2 - Quantification of immunogold labeling on ultrathin sections of HeLa cells embedded in Unicryl or in LR White. The labeling density is expressed as number of particles $/ \mu \mathrm{m}^{2}$ \pm SD. Uni-T - Unicryl, polymerization at $50^{\circ} \mathrm{C}$; Uni-UV Unicryl, polymerization at $4^{\circ} \mathrm{C}$ under UV light; LR-T - LR White, polymerization at $50^{\circ} \mathrm{C}$; LR-UV - LR White, polymerization at $4^{\circ} \mathrm{C}$ under UV light. A, labeling density of Sm antigen; B, density of background labeling. 
Table I

Statistical evaluation of differences in Sm labeling density. Uni-T - Unicryl, polymerization at $50^{\circ} \mathrm{C}$; Uni-UV - Unicryl, polymerization at $4^{\circ} \mathrm{C}$ under UV light; LR-T - LR White, polymerization at $50^{\circ} \mathrm{C}$; LR-UV - LR White, polymerization at $4^{\circ} \mathrm{C}$ under UV light. NS - non-significant ( $\left.\mathrm{p} \geq 0.05\right), * *-\mathrm{p}<0.01$

\begin{tabular}{lcccc}
\hline & Uni-T & Uni-UV & LR-T & LR-UV \\
\hline Uni-T & & NS & $* *$ & $* *$ \\
Uni-UV & NS & $* *$ & $* *$ & $* *$ \\
LR-T & $* *$ & $* *$ & $* *$ \\
LR-UV & $* *$ & $* *$ & $* *$ & \\
\hline
\end{tabular}

Hozak et al., 1994b, Nowak et al., 1997). Unicryl embedding, however, showed worse ultrastructural preservation, especially for the cell nuclei of HeLa cells. This comes into contradiction with previous studies using Unicryl, where it was especially appreciated for the preservation of structural integrity (Scala et al., 1992; Goping et al., 1996; Bogers et al., 1996; Gonzalez Santander et al., 1997). These discrepancies may be accounted for differences in protocols used. Goping and co-workers (1996) applied strong fixation with $2.2 \%$ glutaraldehyde. When $4 \%$ paraformaldehyde with $0.2 \%$ glutaraldehyde was used, Unicryl caused considerable shrinkage and deformation of the cells in their studies. Gonzalez Santander and co-workers (1997) used even stronger fixation with glutaraldehyde-tannic acid/osmium tetroxide. Bogers and co-authors (1996) obtained good ultrastructural results after fixation with $4 \%$ paraformaldehyde and $0.2 \%$ glutaraldehyde, which is still higher than in our protocol, followed by infiltration and resin polymerization at $-20^{\circ} \mathrm{C}$. However, Scala and co-workers (1992), who originally formulated the Unicryl resin, used also quite gentle fixation (4\% paraformaldehyde and $0.1 \%$ glutaraldehyde), processing at room temperature, and polymerization at $4^{\circ} \mathrm{C}$ by UV light. They show very good structure of cytoplasm in human pancreatic cells but do not mention nuclear morphology. It is possible that the differences are due to longer fixation (3 hours in their protocol). On the other hand, Slater and Mason (1994) report inferior ultrastructural performance of Unicryl compared to LR White, which is in agreement with our results. In general, it seems that Unicryl possesses stronger extracting properties than LR White, and that this feature becomes prominent on delicately fixed specimens. This can also explain higher labeling density that we obtained on LR White-embedded cells. Goping et al. (1996) show higher labeling efficiency for Unicryl than for LR White after strong glutaraldehyde fixation. The antigens in their study (dophamine $\beta$-hydroxylase, tyrosine hydroxylase, and the membrane channel protein annexin VII) were obviously aldehyde-fixation insensitive. In contrast, for many nuclear antigens it is crucial to use mild fixation with $4 \%$ formaldehyde and $0.1-0.2 \%$ glutaraldehyde as a maximum.

Background labeling in our study was negligible in all samples. However, the ratio between total labeling density and background labeling density was somewhat higher for Unicryl, and for both resins this value was better after UV-polymeriza-

Table II

Ratio of the total label density to the background label density. Uni-T - Unicryl, polymerization at $50^{\circ} \mathrm{C}$; LR-T - LR White, polymerization at $50^{\circ} \mathrm{C}$; Uni-UV - Unicryl, polymerization at $4^{\circ} \mathrm{C}$ under UV light; LR-UV - LR White, polymerization at $4^{\circ} \mathrm{C}$ under UV light

\begin{tabular}{lcccc}
\hline Sample & Uni-T & LR-T & Uni-UV & LR-UV \\
\hline $\begin{array}{l}\text { Total labeling density/ } \\
\text { background labeling density }\end{array}$ & 93.6 & 80.0 & 154.8 & 135.6 \\
\hline
\end{tabular}


tion than after heat polymerization. For detection of such an abundant antigen as $\mathrm{Sm}$, this characteristic is not of great importance. Nevertheless, in a case of a weak overall labeling (little amount of antigen, low affinity of the antibody) it is crucial to have larger difference between background and specific labeling. In this case, Unicryl can offer certain advantages; however, only for aldehyde-insensitive antigens. Whatever resin is used, the UV-polymerization results in more specific signal than the thermal polymerisation.

On the basis of presented results, several recommendations can be made: 1), for routine immunolocalization studies of nuclear antigens requiring mild fixation and simple embedding protocol, LR White is generally advantageous over Unicryl; 2), when the overall labeling for an antigen of interest is very low, Unicryl may be chosen for having better total label/background ratio. However, fixation and embedding procedure should be then modified to improve ultrastructural preservation; 3 ), polymerization at $4^{\circ} \mathrm{C}$ under UV light is preferable to thermal polymerization.

\section{ACKNOWLEDGEMENTS}

This work was supported by the Grant Agency of the Academy of Sciences of the Czech Republic (Reg.No. A5039701), by the Grant Agency of the Czech Republic (Reg.No. 304/01/0661), and by the institutional grant (No. AV0Z5039906).

\section{REFERENCES}

Bogers J.J., Nibbeling H.A., Deelder A.M., and van Marck E.A.: Quantitative and morphological aspects of Unicryl versus Lowicryl K4M embedding in immunoelectron microscopic studies. J. Histochem. Cytochem. 44, 43-8, 1996.

Gonzalez Santander R., Martinez Cuadrado G., Gonzalez-Santander Martinez M., Monteagudo M., Martinez Alonso F.J., and Toledo Lobo M.V.: The use of different fixatives and hydrophilic embedding media (Historesin and Unicryl) for the study of embryonic tissues. Microsc. Res. Tech. 36, 151-8, 1997.

Goping G., Kuijpers G.A., Vinet R., and Pollard H.B.: Comparison of LR White and Unicryl as embedding media for light and electron immunomicroscopy of chromaffin cells. J. Histochem. Cytochem. 44, 289-95, 1996.

Hozak P., Cook P.R., Schofer C., Mosgoller W., and Wachtler F.: Site of transcription of ribosomal RNA and intranucleolar structure in HeLa cells. J. Cell. Sci. 107 (Pt 2), 639-48, 1994a.
Hozak P., Jackson D.A., and Cook P.R.: Replication factories and nuclear bodies: the ultrastructural characterization of replication sites during the cell cycle. J. Cell. Sci. 107 (Pt 8), 2191-202, 1994b.

Miller H.R.: Fixation and tissue preservation for antibody studies. Histochem. J. 4, 305-20, 1972.

Newman G.R.: LR White embedding for immunoelectron microscopy. Histochem. J. 31, 79, 1999.

Newman G.R., and Hobot J.A.: Modern acrylics for postembedding immunostaining techniques. J. Histochem. Cytochem. 35, 971-81, 1987.

Nowak G., Pestic-Dragovich L., Hozak P., Philimonenko A., Simerly C., Schatten G., et al.: Evidence for the presence of myosin I in the nucleus. J. Biol. Chem. 272, 17176-81, 1997.

Scala C., Cenacchi G., Ferrari C., Pasquinelli G., Preda P., and Manara G.C.: A new acrylic resin formulation: a useful tool for histological, ultrastructural, and immunocytochemical investigations. J Histochem Cytochem. 40, 1799-804, 1992.

Seto A.G., Zaug A.J., Sobel S.G., Wolin S.L., and Cech T.R.: Saccharomyces cerevisiae telomerase is an Sm small nuclear ribonucleoprotein particle. Nature 401, 177-80, 1999.

Slater M., and Mason R.S.: Immunogold localization of TGF beta 1 protein and mRNA in human skin using a colloidal gold/digoxygenin system. Histochemistry 102, 153-163, 1994.

Smit J.W., Meijer C.J., Decary F., and Feltkamp-Vroom T.M.: Paraformaldehyde fixation in immunofluorescence and immunoelectron microscopy. Preservation of tissue and cell surface membrane antigens. J. Immunol. Methods 6, 93-8, 1974.

Stirling J.W.: Immuno- and affinity probes for electron microscopy: a review of labeling and preparation techniques. J Histochem Cytochem. 38, 145-57, 1990.

Tokuyasu K.T.: Immuno-cryoultramicrotomy. In Immunolabeling for electron microscopy. (Eds Polak, J.M., Varndell, I.M.), Elsevier Science Publishers, Amsterdam, New York, Oxford, pp. 71-96, 1984.

Will C.L., and Luhrmann R. Protein functions in pre-mRNA splicing. Curr. Opin. Cell Biol. 9, 320-8, 1997. 